\title{
The changing trends of HIV-1 prevalence and incidence from sentinel surveillance of five sub-populations in Yunnan, China, 2001-2010
}

Li Yang ${ }^{\dagger}$, Min Chen ${ }^{\dagger}$, Yanling Ma, Hongbing Luo, Chaojun Yang, Yingzhen Su, Huichao Chen, Yuhua Shi, Jingyuan Mei, Manhong Jia* and Lin Lu*

\begin{abstract}
Background: Yunnan is one of the provinces hardest-hit by HIV in China. To understand HIV epidemic dynamics and evaluate prevention effectiveness, we studied the changing trends in HIV-1 prevalence and incidence among five sub-populations in Yunnan.

Methods: Consecutive sentinel surveillances were conducted among people who inject drugs (PWID), male sexually transmitted diseases (STD) clinic attendees, and pregnant women for 2001-2010,female sex workers (FSWs) for 2007-2010, men who have sex with men (MSM) for 2008-2010. For the newly diagnosed HIV-seropositive samples, the recent infections were determined with BED-capture enzyme immunoassay (BED-CEIA), based on which HIV incidence was calculated for each sub-population using McDougal algorithm.

Results: From 231,117 individuals, 6,107 HIV-positive samples were tested with BED-CEIA, among which 964 samples were identified as recent infections. In PWID, HIV prevalence for 2001-2010 was between $27.16 \%$ and $18.35 \%$, while the estimated incidence rate significantly decreased from $11.68 \%$ in 2001 to $1.70 \%$ in 2010. Among male STD clinic attendees, both the HIV prevalence (from 3.62\% in 2001 to 1.73\% in 2010) and incidence (from 1.10\% in 2001 to $0.40 \%$ in 2010) showed a significant decreasing trend. In FSWs, the HIV prevalence for 2007-2010 kept stable (between $2.46 \%$ and $1.95 \%$ ), while the HIV incidence significantly decreased (from $0.71 \%$ in 2007 to $0.31 \%$ in 2010). In MSM, the HIV prevalence (between 11.78\% and 9.42\%) and incidence (between $6.01 \%$ and $8.38 \%$ ) remained stable at a relatively high level for 2008-2010. In pregnant women, the HIV prevalence (between 0.44\% and 0.30\%) and incidence (between $0.15 \%$ and $0.08 \%$ ) remained stable for 2001-2010.
\end{abstract}

Conclusion: The HIV incidences in PWID, male STD clinic attendees and FSWs showed the decreasing trend, supporting a positive effect of prevention strategies for these sub-populations. MSM with the highest HIV incidence have become the sub-population most at risk. In most sub-populations, the HIV prevalence did not decline, suggesting the disease burden is still heavy. These findings are valuable for developing HIV prevention strategies in Yunnan.

Keywords: Human Immunodeficiency Virus-1 (HIV-1), People who inject drugs (PWID), Male STD clinic attendees, Female Sex Workers (FSWs), Men who have sex with men (MSM), Pregnant Women, Prevalence, Incidence, Yunnan, China

\footnotetext{
* Correspondence: jiamanhong@hotmail.com; lulin@yncdc.cn

${ }^{\dagger}$ Equal contributors

Institute for STD/AIDS Control and Prevention, Yunnan Center for Disease

Control and Prevention, No 158, Dongsi Street, Xishan District, Kunming

650022, Yunnan Province, China
}

\section{() Biomed Central}

(C) 2015 Yang et al.; licensee BioMed Central. This is an Open Access article distributed under the terms of the Creative Commons Attribution License (http://creativecommons.org/licenses/by/4.0), which permits unrestricted use, distribution, and reproduction in any medium, provided the original work is properly credited. The Creative Commons Public Domain Dedication waiver (http://creativecommons.org/publicdomain/zero/1.0/) applies to the data made available in this article unless otherwise stated. 


\section{Background}

Yunnan is located in Southwest China and situated along the drug trafficking routes channeling heroin into China from Southeast Asia's opium-producing "Golden Triangle" region [1]. Since the first HIV epidemic in China was identified among people who inject drugs (PWID) in Yunnan in 1989, Yunnan has been one of the areas hardest hit by HIV in China [2]. By the end of 2010, the cumulative number of reported HIV infectors and AIDS patients (HIV/AIDS) in Yunnan was 83,925, accounting for $21.0 \%$ of the total national figure [3]. Furthermore, Yunnan serves as a primary entry point for the introduction of different HIV-1 genotypes into China [4-6]. Thus, Yunnan is accepted as an epicenter of the HIV-1 epidemic in China [2,7].

Usually, prevalence and incidence are used to describe the characteristic of HIV epidemic in a given population. Prevalence is the number of people living with HIV infection at a given time, including recent and long-term infections. Prevalence is most useful for measuring the burden of HIV in a population. However, incidence is the number of new HIV infections that occur during a given year. HIV incidence reflects the level of on-going HIV transmission, HIV infection trends, and the impact of HIV prevention efforts. Traditionally, HIV incidence estimation can be obtained through a prospective cohort study, in which participants are repeatedly tested. However, such surveillances are difficult to establish and costly to maintain. And cohort studies cannot avoid some of the biases inherent, such as the loss to followup and the change of participant behavior following riskreduction counseling [8]. Alternatively, laboratory-based incidence assays have been developed since 1998 [9,10]. Among these methods, the BED-capture enzyme immunoassay (BED-CEIA) was the most commonly used incidence assay, which is based on measurement of the proportion of HIV-1-specific IgG to total IgG after seroconversion [11]. After the introduction of BED-CEIA into China in 2005, the correction factors were reset based on the characteristics of Chinese population [12]. The related studies showed that the HIV-1 incidence estimates yielded by BED-CEIA were similar to those obtained by cohort studies in China [12-14]. Yunnan was one of the provinces that earlier adopted BED-CEIA for HIV-1 incidence surveillance.

In China, the national comprehensive surveillance for HIV epidemic includes the national HIV sentinel surveillance system, HIV/AIDS case reporting system, and special epidemiologic surveys $[15,16]$. HIV sentinel surveillance is a series of cross-sectional surveys on risk behaviors and sero-testing in the representative areas and populations. The data from sentinel surveillance are used to analyze the trend of HIV prevalence and the risk factors for HIV infection in a giving region and population. The national sentinel surveillance sites have been established since 1995 [16]. With development, the national sentinel surveillance sites covers STD clinic attendees, female sexual workers (FSWs), PWID, long-distance truck drivers, pregnant women, paid blood donors, men who have sex with men (MSM), clients of female sex workers and tuberculosis (TB) patients $[15,16]$. To improve the coverage, some provincial sentinel surveillance sites were also established. These sentinel surveillance sites improved the ability to evaluate the HIV epidemic in China. Otherwise, the web-directed HIV/ AIDS case reporting system started to run from March 2005. According to the regulation for HIV/AIDS cases reporting, all newly identified HIV/AIDS cases by confirmatory HIV testing are required to be reported into the system within 24 hours.

However, the present surveillance can not directly provide the HIV incidence, which is vital to understand HIV transmission dynamics and evaluate prevention effectiveness. To improve the utilization of the data, the combination of epidemiological surveillance and laboratory assay may be a good choice. In this study, BED-CEIA was used to detect recent infection with the samples from sentinel surveillance for 2001-2010 in Yunnan, including PWID, male STD clinic attendees, FSWs, MSM and pregnant women. This study explored the HIV prevalence and incidence trends from 2001 to 2010 in Yunnan, and is important to direct the future prevention and control efforts.

\section{Methods}

\section{Sample collection and serological testing}

By 2010, 90 sentinel sites were set up for regular surveillance among PWID (24 Sites; sample size: 400 per site), male STD clinic attendees (15 Sites; sample size: 400 per site), pregnant women (15 Sites; sample size: 400 per site), FSWs (32 Sites; sample size: 400 per site), and MSM (4 Sites; sample size: 200 per site) in Yunnan Province. Consecutive sentinel surveillances were conducted each year among PWID, male STD clinic attendees and pregnant women between 2001 and 2010, among FSWs between 2007 and 2010, among MSM between 2008 and 2010. Each participant received an anonymous interview to collect information on demographic details and HIV transmission related risk behaviors and provided 2-3 ml of whole blood. Plasma was separated from whole blood for HIV testing. Samples were screened for HIV-1 antibody with an enzyme immunoassay or rapid test, and then HIV-1 infection status was confirmed with a Western blot assay (HIV BLOT 2.2, MP Diagnostics, Singapore). All HIV tests were informed and voluntary. Written consents were obtained from all participants. The study was approved by Biomedical Ethics Review Committee of Yunnan Province. 


\section{BED capture enzyme immunoassay}

Excluding previously reported HIV-seropositive samples, the newly diagnosed HIV-seropositive samples were subjected to BED-CEIA testing. The BED-CEIA was performed according to the manufacturer's instructions (Calypte HIV-1 BED incidence EIA, Calypte Biomedical Corporation, Portland, OR). Appropriate calibrator and negative control, low positive control and high positive control were run in triplicate for every plate. Test specimens were initially run singly. The optical density (OD) values of test specimens were normalized by a ratio using a calibrator (specimen OD/calibrator $\mathrm{OD)}$ to minimize inter-run variations. If the normalized OD (ODn) was $>1.2$, the specimen was classified as being from a long-term seroconverter. Specimens with ODn $<1.2$ were tested again in triplicate to confirm the values. In confirmatory testing, specimens with ODn values $<0.8$ were considered to have undergone recent seroconversion.

\section{Calculation of incidence}

HIV incidence rate was calculated using McDougal formula [17]:

$$
I=\frac{F \times(365 / w) \times R}{N+F \times(365 / w) \times R / 2} \times 100 \%
$$

Where $I$ is the annual HIV-1 incidence; $F$ is the correction factor; $w$ is the window period (the mean length of time individuals remain classified as "recently infected" by BED-CEIA; in China, $w=168$ days) [12]; $R$ is the number of recent HIV infections detected by BEDCEIA; $\mathrm{N}$ is the total number of HIV-seronegative subjects.

The correct factor was calculated using the following formula [17]:

$$
F=\frac{(R / P)+\gamma-1}{(R / P) \times(a-\beta+2 \gamma-1)} \times 100 \%
$$

Where $R$ is the number of recent HIV infections detected by BED-CEIA; $P$ is the total number of HIVseropositive subjects that should be detected with BEDCEIA; $\alpha$ is the sensitivity of BED-CEIA for recent infection ( $<1$ week); $\beta$ is the specificity of BED-CEIA for the sample whose infected time between 1 week and 2 weeks; $\gamma$ is the specificity of BED-CEIA for the sample whose infected time beyond 2 weeks. In China, $\alpha=0.8098, \beta=0.7571$ and $\gamma=0.9315$ [12].

When only a part of samples are detected by BEDCEIA among all the samples that should be detected, the number of recent HIV infections is adjusted using the following formula: $R=R^{\prime} \times\left(P^{\prime} / P\right)$. Where $R^{\prime}$ is the number of recent HIV infections actually determined by BEDCEIA, $\mathrm{P}^{\prime}$ is the number of HIV-seropositive samples actually detected with BED-CEIA, $p$ is the number of HIV-seropositive subjects that should be detected with BED-CEIA.

The 95\% confidence interval (CI) for the incidence estimate is given by the following formula:

$$
95 \% \mathrm{CI}=\frac{ \pm 1.96 I}{\sqrt{R}}
$$

According to the Operations Manual for HIV-1 Recent Infections Surveillance issued by Chinese Center for Disease Control and Prevention, the estimated incidence is unreliable if the number of recent infections identified by BED-CEIA is less than 10. For pregnant women, the number of recent infections for each year was less than 10, a solution was to combine the data of every two years to calculate the incidence. For the other four sub-populations, the incidences for each year were calculated.

\section{Statistical analysis}

Statistical analyses were conducted using the SPSS 19.0 statistical analysis software package (SPSS Inc. Chicago, IL). Categorical variables were compared using $x^{2}$ tests. Trend tests were performed using $X^{2}$ tests with linearby-linear association. All tests were two-tailed and a $p$ value $<0.05$ was considered statistically significant.

\section{Results}

A total of 231,117 subjects were tested for HIV in sentinel surveillance program between 2001 and 2010, including 25,654 PWID, 48,125 male STD clinic attendees, 110,126 pregnant women, 45,604 FSWs (2007-2010) and 1,611 MSM (2008-2010). Through serum screening, HIV-1 antibodies were identified in 8,432 samples, in which 1,991 subjects had been reported as HIV-1 infection before participating in the sentinel surveillance program. All the newly reported HIV-1 infected subjects were confirmed with western blot assay. Except 334 samples whose volumes were not enough for BEDCEIA, a total of 6,107 samples (94.8\%) were tested with BED-CEIA, among which 964 samples were identified as HIV-1 recent infection. Based on these, the prevalence and incidence estimates were obtained for each of the five sub-populations (Table 1).

\section{PWID}

The HIV prevalence and the estimated HIV incidence from 2001 to 2010 among PWID were shown in Table 1. The HIV prevalence did not show a decreasing trend (trend test, $\mathrm{p}=0.051$ ). However, the HIV incidence showed a significant decreasing trend for 2001-2010 (trend test, $\mathrm{p}<0.01$ ). 
Table 1 HIV-1 prevalence and incidence from sentinel surveillance

\begin{tabular}{|c|c|c|c|c|c|c|c|c|}
\hline Population & Year & $\begin{array}{l}\text { The number } \\
\text { of subjects }\end{array}$ & $\begin{array}{l}\text { The number of HIV } \\
\text { antibody-positive } \\
\text { subjects }\end{array}$ & $\begin{array}{l}\text { Prevalence } \\
\text { rate }(\%, 95 \% \mathrm{Cl})\end{array}$ & $\begin{array}{l}\text { The number of } \\
\text { previously } \\
\text { reported cases }\end{array}$ & $\begin{array}{l}\text { The number of } \\
\text { samples tested } \\
\text { with BED-CEIA }\end{array}$ & $\begin{array}{l}\text { The number of } \\
\text { recently infected } \\
\text { individuals }\end{array}$ & $\begin{array}{l}\text { Estimated HIV } \\
\text { incidence rate } \\
(\%, 95 \% \mathrm{Cl})\end{array}$ \\
\hline \multirow[t]{10}{*}{ PWID } & 2001 & 1344 & 365 & $27.16(24.37 \sim 29.94)$ & 0 & 345 & 72 & $11.68(9.06 \sim 14.30)$ \\
\hline & 2002 & 1651 & 379 & $22.96(20.64 \sim 25.27)$ & 0 & 378 & 100 & $12.96(10.42 \sim 15.50)$ \\
\hline & 2003 & 2212 & 475 & $21.47(19.54 \sim 23.40)$ & 9 & 464 & 52 & $2.74(1.99 \sim 3.48)$ \\
\hline & 2004 & 2331 & 531 & $22.78(20.84 \sim 24.72)$ & 12 & 517 & 76 & $5.23(4.06 \sim 6.40)$ \\
\hline & 2005 & 3008 & 561 & 18.65 (17.11 20.19) & 33 & 503 & 55 & $2.07(1.54 \sim 2.60)$ \\
\hline & 2006 & 2338 & 491 & $21.00(19.14 \sim 22.86)$ & 171 & 284 & 32 & $1.80(1.21 \sim 2.39)$ \\
\hline & 2007 & 2416 & 571 & $23.63(21.70 \sim 25.57)$ & 223 & 331 & 39 & $2.18(1.52 \sim 2.85)$ \\
\hline & 2008 & 2691 & 563 & 20.92 (19.19 22.65) & 162 & 338 & 38 & $1.94(1.38 \sim 2.51)$ \\
\hline & 2009 & 4626 & 1314 & 28.40 (26.87 29.94) & 717 & 586 & 66 & 1.87 (1.42 2.32) \\
\hline & 2010 & 3037 & 633 & $20.84(19.22 \sim 22.47)$ & 318 & 307 & 38 & $1.70(1.17 \sim 2.24)$ \\
\hline \multirow[t]{10}{*}{ Male STD clinic attendees } & 2001 & 3922 & 142 & $3.62(3.03 \sim 4.22)$ & 0 & 140 & 27 & $1.10(0.69 \sim 1.52)$ \\
\hline & 2002 & 5222 & 122 & $2.34(1.92 \sim 2.75)$ & 0 & 120 & 27 & $0.88(0.55 \sim 1.22)$ \\
\hline & 2003 & 5419 & 135 & $2.49(2.07 \sim 2.91)$ & 0 & 123 & 18 & $0.47(0.26 \sim 0.68)$ \\
\hline & 2004 & 5627 & 113 & $2.01(1.64 \sim 2.38)$ & 3 & 101 & 18 & $0.52(0.29 \sim 0.75)$ \\
\hline & 2005 & 5611 & 89 & $1.59(1.26 \sim 1.92)$ & 0 & 85 & 15 & $0.41(0.21 \sim 0.62)$ \\
\hline & 2006 & 5636 & 88 & $1.56(1.24 \sim 1.89)$ & 0 & 60 & 10 & $0.37(0.18 \sim 0.56)$ \\
\hline & 2007 & 4989 & 83 & $1.66(1.31 \sim 2.02)$ & 5 & 76 & 10 & $0.24(0.09 \sim 0.38)$ \\
\hline & 2008 & 4956 & 67 & $1.35(1.03 \sim 1.68)$ & 5 & 55 & 8 & $0.23(0.08 \sim 0.38)$ \\
\hline & 2009 & 3731 & 50 & $1.34(0.97 \sim 1.71)$ & 23 & 26 & 8 & $0.42(0.13 \sim 0.70)$ \\
\hline & 2010 & 3012 & 52 & $1.73(1.26 \sim 2.20)$ & 19 & 32 & 7 & $0.40(0.11 \sim 0.69)$ \\
\hline \multirow[t]{4}{*}{ FSWs } & 2007 & 11775 & 290 & $2.46(2.18 \sim 2.75)$ & 21 & 255 & 50 & $0.71(0.52 \sim 0.90)$ \\
\hline & 2008 & 11604 & 226 & $1.95(1.69 \sim 2.20)$ & 12 & 202 & 32 & $0.40(0.27 \sim 0.54)$ \\
\hline & 2009 & 12465 & 276 & $2.21(1.95 \sim 2.48)$ & 125 & 140 & 25 & $0.32(0.20 \sim 0.44)$ \\
\hline & 2010 & 9760 & 217 & $2.22(1.93 \sim 2.52)$ & 105 & 112 & 20 & $0.31(0.17 \sim 0.44)$ \\
\hline \multirow[t]{3}{*}{ MSM } & 2008 & 450 & 53 & 11.78 (8.61 14.95) & 0 & 53 & 14 & $6.01(2.86 \sim 9.16)$ \\
\hline & 2009 & 467 & 44 & $9.42(6.64 \sim 12.21)$ & 2 & 42 & 15 & $6.58(3.25 \sim 9.90)$ \\
\hline & 2010 & 694 & 71 & $10.23(7.85 \sim 12.61)$ & 12 & 59 & 27 & $8.38(5.22 \sim 11.54)$ \\
\hline
\end{tabular}


Table 1 HIV-1 prevalence and incidence from sentinel surveillance (Continued)

\begin{tabular}{|c|c|c|c|c|c|c|c|c|}
\hline \multirow[t]{5}{*}{ Pregnant women } & 2001-2002 & 20032 & 60 & $0.30(0.22 \sim 0.38)$ & 0 & 53 & 10 & $0.09(0.04 \sim 0.14)$ \\
\hline & 2003-2004 & 23254 & 102 & $0.44(0.35 \sim 0.52)$ & 1 & 94 & 15 & $0.09(0.05 \sim 0.14)$ \\
\hline & $2005-2006$ & 23795 & 102 & $0.43(0.35 \sim 0.51)$ & 0 & 87 & 13 & $0.08(0.04 \sim 0.12)$ \\
\hline & 2007-2008 & 26010 & 108 & $0.42(0.34 \sim 0.49)$ & 1 & 98 & 15 & $0.08(0.04 \sim 0.12)$ \\
\hline & 2009-2010 & 17032 & 59 & $0.35(0.26 \sim 0.43)$ & 12 & 41 & 12 & $0.15(0.07 \sim 0.22)$ \\
\hline
\end{tabular}




\section{Male STD clinic attendees}

For male STD clinic attendees, the HIV prevalence and the estimated HIV incidence were shown in Table 1. Both the HIV prevalence and incidence among male STD clinic attendees showed a significant decreasing trend for 2001-2010 (trend test, $\mathrm{p}<0.01$ ).

\section{FSWs}

In 2007, 14 additional FSWs sentinel surveillance sites were established, which improved monitoring HIV epidemic among this sub-population in Yunnan [7]. Thus, the FSWs surveillance data from 2007 to 2010 were used for analysis. From 2007 to 2010, the HIV prevalence and the estimated HIV incidence in FSWs were shown in Table 1. The HIV prevalence in FSWs kept stable (trend test, $\mathrm{p}=0.431$ ). However, the HIV incidence in FSWs showed a significant decreasing trend for 2001-2010 (trend test, $\mathrm{p}<0.01$ ).

\section{MSM}

Province-wide MSM sentinel surveillance initiated in 2008. From 2008 to 2010, the HIV prevalence and the estimated HIV incidence in MSM were shown in Table 1. The HIV prevalence and incidence in MSM showed no significant change and remained stable at a relatively high level for 2008-2010 (trend test, $\mathrm{p}=0.492$ and $\mathrm{p}=$ $0.165)$.

\section{Pregnant women}

For pregnant women, the surveillance data of every two years were merged to estimate the incidence from 2001 to 2010, the HIV prevalence and the estimated HIV incidence in pregnant women were shown in Table 1 . The HIV prevalence and incidence in pregnant women remained stable for 2001-2010 (trend test, $\mathrm{p}=0.552$ and $\mathrm{p}=0.190)$.

\section{Discussion}

In this work, we first reported the province-wide application of BED-CEIA to estimate HIV-1 incidence among five sub-populations in China. To our knowledge, the study period of this work is the longest, and the sample size is the largest in China, which includes 10-year sentinel surveillance data and provides the trends of HIV-1 prevalence and incidence among five sub-populations for 2001-2010 in Yunnan, one of the provinces hardesthit by HIV in China. The finding of this work is important to understand HIV-1 epidemic and evaluate the measures of HIV prevention and control in the first 10 years of this century in Yunnan.

In 1989, the first HIV epidemic in Yunnan was identified among PWID in Ruili County bordering Myanmar. Since then, PWID had become the prime force to drive the development of HIV epidemic in Yunnan [2,7].
Surveillance data showed that the average prevalence among PWID increased from 1992 to 1999 (2.7\% in 1992, $15.0 \%$ in 1995 and $30.0 \%$ in 1999) [7]. The data from reported cases showed that the intravenous injection had been the premier transmission route until 2006 $[2,7]$. In this study, we found that the estimated HIV incidence rate among PWID remained around 12\% from 2001 to 2002. In 2003, it significantly decreased. After 2005 , it remained near $2 \%$. The significant decrease of HIV incidence rate among PWID could result from the implement of the needle exchange programs (NEPs, introduced in 2002) and methadone maintenance treatment (MMT, introduced in 2004) [18-20]. Based on a mathematical transmission model, it was estimated that NEPs in Yunnan have averted approximately 16-20\% (5,200-7,500 infections) of the expected HIV cases from 2002 to 2008 [21]. However, the prevalence among PWID remained stable at a high level during the study period, and was the highest among four high-risk groups. Moreover, HIV prevalence among PWID in Yunnan was two times of the national average level in this population for 2001-2010 [3]. The life cycle of PWID extended with the introduction of a nationally subsidized antiretroviral therapy program in 2004. Thus, the infected PWID would be repeatedly recruited in the sentinel surveillance. Meanwhile, more previously undetected infections were found with the expanding HIV testing in Yunnan. All of these could attenuate the contribution of the incidence decreasing.

A part of male STD clinic attendees were reported to visit FSWs [22]. As the important bridging populations, FSWs and male STD clinic attendees played an important role in driving sexual transmission and the transition of the epidemic from high-risk population to general population. Because of the limitation of surveillance data, the estimation of HIV epidemic in FSWs was performed only from 2007 to 2010 in this study. Nevertheless, the both HIV incidence rates among FSWs and male STD clinic attendees showed decreasing trend, which may related with the promotion of condom use and health education [23], which was introduced in 2002. According to the data of FSWs integrated intervention in Yunnan, HIV knowledge awareness rate increased from $82 \%$ in 2007 to $95 \%$ in 2010 . More FSWs consistently used condom with clients in the last month (75\% in 2007; and $86 \%$ in 2010) and in the last commercial sex (92\% in 2007; and 94\% in 2010). Male STD clinic attendees were the only population in which the HIV prevalence significantly decreased. Besides the decrease of incidence rate, there are other possible reasons. Because most STDs are curable, some STD clinic attendees infected with HIV would not return to STD clinic after their STDs were cured. This part of individuals would not be monitored in the following years, which means 
some long-term infectors were excluded when calculating the prevalence using the data from the sentinel sites of Male STD clinic attendees.

In recent years, a fast-spreading HIV epidemic among MSM brings a new challenge to China. The national HIV prevalence from MSM sentinel surveillance data showed a rising trend from $0.9 \%$ in 2003 to $6.3 \%$ in 2011 [24]. In this study, we found HIV prevalence from MSM sentinel surveillance in Yunnan was about 10\% from 2008 to 2010, which suggested that Yunnan bears higher HIV burden in the MSM population. Strikingly, the HIV incidence in MSM was the highest among the five subpopulations and reached $8.38 \%$ in 2010 . Although the HIV prevalence and incidence in MSM showed no significant change for 2008-2010, based on the HIV/AIDS case reporting system, the annual proportion of newly reported HIV cases attributed to homosexually transmitted infection in Yunnan increased $(6.1 \%$ in 2008 , 9.1\% in 2009 and $12.0 \%$ in 2010), which may result from scaling up HIV testing among this population. Usually, MSM are difficult to access because of their underground behaviors and mobile lifestyles. From 2007, the intervention provided by peer educators from community based organizations was introduced, which promotes HIV testing, active care and antiretroviral therapy of HIVpositive MSM [25]. Presently, the epidemic in MSM is in the ascending period, more attention should be given to this sub-population.

HIV prevalence in pregnant women can be representative of that in the general population [26]. The data from pregnant women had been used to compare the regional variation of HIV epidemic [27,28]. Our study found that the HIV prevalence and incidence in pregnant women showed no significant change for 2001-2010, which suggested that HIV transmission between spouses and sexual partners was relatively stable.

According to the data of reported cases, the main transmission route changed from intravenous injection to sexual contact after 2006 [7]. According to the HIV/ AIDS case reporting system, among the annual newly reported HIV cases, the proportion of the individuals infected by heterosexual contact continually increased from $30.6 \%$ in 2006 to $58.4 \%$ in 2010 . However, the increase of the proportion of the sexual transmission did not convince the decreasing incidence observed in sentinel surveillance from the heterosexual transmission related populations, such as FSWs and male STD clinic attendees. The possible explanation may be that the HIV epidemic has transited from the high-risk populations to the general population, in which non-commercial sexual behaviors might be more prevalent than commercial sexual behaviors. Because the scale of the general population is larger than that of the high-risk populations, even if the incidence is low, the number of HIV-infected individuals will be larger. Furthermore, the more HIVinfected individuals previously undetected were found with increasing HIV testing(The total annual number for HIV testing increased from 95,755 in 2001 to 2,766,106 in 2010). Thus, the recent infection research for annual reported cases may provide more information to evaluate the epidemic in general population.

In fact, when using BED-CEIA, the misclassification of established infections as recently acquired infections may occur due to false-positive tests resulting from various reasons, such as antiretroviral therapy, low $\mathrm{CD} 4^{+} \mathrm{T}$ lymphocytes counting and HIV-1 genotypes [29]. To correct the potential disturbance, two strategies have been adopted. One is case-based adjustment, to exclude long-term infections by using additional information, such as the recorder of previous HIV testing and diagnosis and the number of $\mathrm{CD}_{4}^{+} \mathrm{T}$ lymphocytes. The other is formula-based adjustment using local correction factors for incidence estimates. In this study, although these two strategies have been considered, the misclassification may not be completely avoided. However, what we really concern is the changing trend of HIV incidence, which directly reflects HIV transmission dynamics and prevention effectiveness. With the consistent standard, the consecutive sentinel surveillances and BED-CEIA testing could provide the trend of HIV incidence.

\section{Conclusions}

In conclusion, our study elucidated the trend of HIV prevalence and incidence for 2001-2010 among five sub-populations in Yunnan. HIV incidences among PWID, male STD clinic attendees and FSWs showed the declining trends, which suggested that the prevention and control efforts for these population should be continued. However, HIV incidence in MSM showed no significant change and remained stable at a relatively high level, which suggested that more resource should be input and the effective measures for HIV prevention should be developed for this population.

\section{Competing interests}

The authors declare that they have no competing interests.

\section{Authors' contributions}

Conceived and designed the experiments: YM MJ LL. Collected the samples and performed the experiments: LY CY YZS HC MC YHS. Collected the epidemiological information: HL JM. Analyzed the data: LY MC YM. Interpretation of results: LY MC YM MJ LL. Wrote the paper: MC. Critically revised manuscript: YM MJ. Approved the final version of this manuscript: $L Y$ MC YM HL CY YZS HC YHS JM MJ LL.

\section{Acknowledgements}

We acknowledge the NIH Fellows Editorial Board for editorial assistance and appreciate the kind assistance provided by Dr. Aijuan Qu (National Institutes of Health, USA). This work was supported by the Major Project of China's "Twelfth Five- Year Plan" for Science and Technology Development (2011ZX10004-903) and (2012ZX10001001-10). 
Received: 20 September 2014 Accepted: 30 March 2015 Published online: 12 April 2015

\section{References}

1. Beyrer C, Razak MH, Lisam K, Chen J, Lui W, Yu XF. Overland heroin trafficking routes and HIV-1 spread in south and south-east Asia. Aids. 2000;14(1):75-83.

2. $L u L$, Jia $M, M a Y, Y$ ang $L$, Chen $Z$, Ho DD, et al. The changing face of HIV in China. Nature. 2008:455(7213):609-11.

3. China Ministry of Health, Joint United Nations Programme on HIV/AIDS, World Health Organization. 2011 update on the HIV/AIDS epidemic in China. Chin J AIDS STD. 2012;18(1):1-5.

4. Graf M, Shao Y, Zhao Q, Seidl T, Kostler J, Wolf H, et al. Cloning and characterization of a virtually full-length HIV type 1 genome from a subtype B'-Thai strain representing the most prevalent B-clade isolate in China. AIDS Res Hum Retroviruses. 1998;14(3):285-8.

5. Luo CC, Tian C, Hu DJ, Kai M, Dondero T, Zheng X. HIV-1 subtype C in China. Lancet. 1995;345(8956):1051-2.

6. Cheng H, Zhang J, Capizzi J, Young NL, Mastro TD. HIV-1 subtype E in Yunnan, China. Lancet. 1994;344(8927):953-4.

7. Jia M, Luo H, Ma Y, Wang N, Smith K, Mei J, et al. The HIV epidemic in Yunnan Province, China, 1989-2007. J Acquir Immune Defic Syndr. 2010;53 Suppl 1:S34-40.

8. Beyrer C, Nelson KE. Loss to follow-up effect in investigations of HIV-1 incidence. Lancet. 1997;349(9052):649-50.

9. Janssen RS, Satten GA, Stramer SL, Rawal BD, O'Brien TR, Weiblen BJ, et al. New testing strategy to detect early HIV-1 infection for use in incidence estimates and for clinical and prevention purposes. JAMA. 1998;280(1):42-8.

10. Busch MP, Pilcher CD, Mastro TD, Kaldor J, Vercauteren G, Rodriguez W, et al. Beyond detuning: 10 years of progress and new challenges in the development and application of assays for HIV incidence estimation. Aids. 2010;24(18):2763-71.

11. Parekh BS, Kennedy MS, Dobbs T, Pau CP, Byers R, Green T, et al. Quantitative detection of increasing HIV type 1 antibodies after seroconversion: a simple assay for detecting recent HIV infection and estimating incidence. AIDS Res Hum Retroviruses. 2002;18(4):295-307.

12. Xiao $Y$, Jiang $Y$, Feng J, Xu W, Wang M, Funkhouser E, et al. Seroincidence of recent human immunodeficiency virus type 1 infections in China. Clin Vaccine Immunol. 2007;14(10):1384-6.

13. Jiang $Y$, Wang $M, N i$ M, Duan S, Wang $Y$, Feng J, et al. HIV-1 incidence estimates using lgG-capture BED-enzyme immunoassay from surveillance sites of injection drug users in three cities of China. Aids. 2007;21 Suppl 8:S47-51.

14. Xu J, Wang H, Jiang Y, Ding G, Jia M, Wang G, et al. Application of the BED capture enzyme immunoassay for HIV incidence estimation among female sex workers in Kaiyuan City, China, 2006-2007. Int J Infect Dis. 2010;14(7):e608-12.

15. Wang L, Wang N. HIV/AIDS epidemic and the development of comprehensive surveillance system in China with challenges. Chin Med J (Engl). 2010;123(23):3495-500.

16. Sun X, Wang N, Li D, Zheng X, Qu S, Wang L, et al. The development of HIV/AIDS surveillance in China. Aids. 2007;21 Suppl 8:S33-8.

17. Centers for Disease Control and Prevention. Interim recommendations for the use of the BED capture enzyme immunoassay for incidence estimation and surveillance. Atlanta, GA: CDC, Office of the Global AIDS Coordinator; 2006.

18. Li J, Ha TH, Zhang C, Liu H. The Chinese government's response to drug use and HIV/AIDS: a review of policies and programs. Harm Reduction J. 2010;7:4.

19. Pang L, Hao Y, Mi G, Wang C, Luo W, Rou K, et al. Effectiveness of first eight methadone maintenance treatment clinics in China. Aids. 2007;21 Suppl 8:S103-7.

20. Yin W, Hao Y, Sun X, Gong X, Li F, Li J, et al. Scaling up the national methadone maintenance treatment program in China: achievements and challenges. Int J Epidemiol. 2010;39 Suppl 2:ii29-37.

21. Zhang L, Yap L, Xun Z, Wu Z, Wilson DP. Needle and syringe programs in Yunnan, China yield health and financial return. BMC Public Health. 2011;11:250.

22. Wang QQ, Chen XS, Yin YP, Liang GJ, Jiang N, Dai T, et al. HIV/STD pattern and its associated risk factors among male STD clinic attendees in China: a foci for HIV intervention. BMC Public Health. 2011;11:955.

23. Ding GW, Hsi JH, Liu HX, Su YY, Wang JJ, Bai J, et al. HIV-infected female sex workers' high risk behavior and attitude changes in Kaiyuan City, Yunnan Province, China. Biomed Environ Sci. 2014;27(6):444-52.
24. Wang L, Norris JL, Li DM, Guo W, Ding ZW, Wang N. HIV prevalence and influencing factors analysis of sentinel surveillance among men who have sex with men in China, 2003-2011. Chin Med J (Engl). 2012;125(11):1857-61.

25. Sullivan PS, Carballo-Dieguez A, Coates T, Goodreau SM, McGowan I, Sanders EJ, et al. Successes and challenges of HIV prevention in men who have sex with men. Lancet. 2012;380(9839):388-99.

26. Grant AD, De Cock KM. The growing challenge of HIV/AIDS in developing countries. Br Med Bull. 1998;54(2):369-81.

27. Tarantola D, Schwartlander B. HIV/AIDS epidemics in sub-Saharan Africa: dynamism, diversity and discrete declines? Aids. 1997;11 Suppl B:S5-21.

28. Quinn TC. Global burden of the HIV pandemic. Lancet. 1996;348(9020):99-106.

29. Parekh BS, Hanson DL, Hargrove J, Branson B, Green T, Dobbs T, et al. Determination of mean recency period for estimation of HIV type 1 Incidence with the BED-capture EIA in persons infected with diverse subtypes. AIDS Res Hum Retroviruses. 2011;27(3):265-73.

\section{Submit your next manuscript to BioMed Central and take full advantage of:}

- Convenient online submission

- Thorough peer review

- No space constraints or color figure charges

- Immediate publication on acceptance

- Inclusion in PubMed, CAS, Scopus and Google Scholar

- Research which is freely available for redistribution 